\title{
Colorimetric detection of ultrathin dielectrics on strong interference coatings
}

\author{
Sencer Ayas, ${ }^{1,3}$ Gokhan Bakan, ${ }^{1,2, *}$ (1) Erol Ozgur, ${ }^{1}$ Kemal Celebi, ${ }^{1}$ Gamze Torunoglu, ${ }^{1}$ and \\ AykutLu Dana ${ }^{1}$ \\ 'UNAM-Institute of Materials Science and Nanotechnology, Bilkent University, Ankara 06800, Turkey \\ ${ }^{2}$ Department of Electrical and Electronics Engineering, Atilim University, Ankara 06830, Turkey \\ ${ }^{3}$ e-mail: ayas@bilkent.edu.tr \\ *Corresponding author: gokhan.bakan@atilim.edu.tr
}

Received 12 January 2018; revised 13 February 2018; accepted 13 February 2018; posted 16 February 2018 (Doc. ID 319524 ); published 14 March 2018

Metal films covered with ultrathin lossy dielectrics can exhibit strong interference effects manifested as the broad absorption of the incident light resulting in distinct surface colors. Despite their simple bilayer structures, such surfaces have only recently been scrutinized and applied mainly to color printing. Here, we report the use of such surfaces for colorimetric detection of ultrathin dielectrics. Upon deposition of a nanometer-thick dielectric on the surface, the absorption peak red shifts, changing the surface color. The color contrast between the bare and dielectric-coated surfaces can be detected by the naked eye. The optical responses of the surfaces are characterized for nanometerthick $\mathrm{SiO}_{2}, \mathrm{Al}_{2} \mathrm{O}_{3}$, and bovine serum albumin molecules. The results suggest that strong interference surfaces can be employed as biosensors. (0)2018 Optical Society of America

OCIS codes: (310.1620) Interference coatings; (310.6188) Spectral properties; (280.4788) Optical sensing and sensors; (260.3910) Metal optics.

https://doi.org/10.1364/OL.43.001379

The interaction of light with nanostructured surfaces offers novel phenomena that cannot be readily available in thin film or bulk optical materials. By the clever design of these surfaces, reflection, transmission, and absorption of the incident light can be controlled, and negative refractive index materials $[1,2]$, perfect light absorbers [3,4], ultrathin monochromatic lenses [5,6], on-chip optical components $[7,8]$ and biosensors $[9,10]$ can be fabricated. In particular, the perfect absorption of light is desired for many optoelectronics and photonic applications such as thermophotovoltaics [11,12], biosensors [13,14], and color printing $[15,16]$. The common approach for designing light absorption in the ultraviolet, visible, and infrared (IR) wavelengths is based on metal/insulator/metal absorbers, also known as plasmonic metamaterial absorbers, which consist of a nanostructured top metal layer [4,17-19]. Alternatively, the use of patternless multilayer thin-film-based metal/dielectric stacks is also possible for color printing and light absorption applications [20]. Reflective color filters [21], tunable resonant surfaces [22,23], and hydrogen sensors [24,25] have been demonstrated using the metal/insulator/metal geometry in the visible wavelengths. Moreover, near perfect light absorption can be achieved by replacing the top metallic layer with an ultrathin lossy dielectric film [26]. If the lossy dielectric film is placed a quarter-wavelength $(t=\lambda / 4 n)$ away from the semicontinuous metal layer, the electric field can be enhanced on the lossy layer, and the light absorption is maximized. Such surfaces have also found their way into enhanced light absorption in the IR wavelengths for radiative cooling [27] and surfaceenhanced IR spectroscopy applications [28]. A recent study by Kats et al. has showed that almost perfect light absorption can be achieved using nanometer-thick lossy dielectric films on semi-continuous metal layers through exhibiting strong interference [29]. These surfaces offer a wide-angle spectral response compared to metal/insulator/metal stacks due to the small phase accumulation in the ultrathin lossy dielectric layer $(t \ll \lambda)$. Moreover, such surfaces exhibit almost perfect absorbance at certain wavelengths, thus having distinctive surface colors depending on the thickness of the lossy dielectric film. Despite the ease of design and fabrication of such surfaces, only a few applications, such as solar water splitting [30], color printing [29,31], and optical data storage [32] have been demonstrated so far. Here, to the best of our knowledge, as a new application, we demonstrate the use of strong interference coatings to detect ultrathin dielectric coatings by the naked eye. The strong interference surfaces used in this Letter consist of $20 \mathrm{~nm}$ thick amorphous $\mathrm{Si}(\mathrm{a}-\mathrm{Si})$ on optically thick Al films [Fig. 1(a)]. The surfaces are fabricated on large areas and exhibit wide-angle and polarization-independent optical responses. The performance of the surfaces is tested by measuring the optical response of the surfaces with atop nanometer-thick lossless dielectrics, monolayers, and bilayers of protein films.

The enhanced absorption by the strong interference surfaces is achieved by almost perfect destructive interference of the reflected rays from the dielectric-air interface $\left[r_{0}\right.$ in Fig. 1(b) $]$ and dielectric-metal interface $\left[r_{1}, r_{2}, \ldots\right.$ in Fig. $\left.1(\mathrm{~b})\right]$. To visualize 
(a)

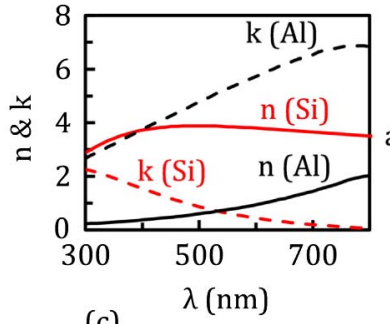

(c)

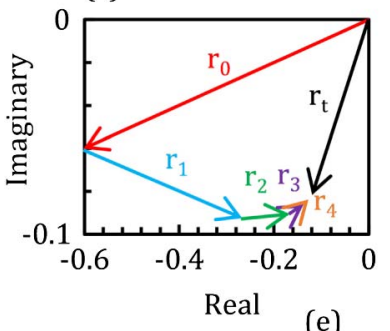

(b)

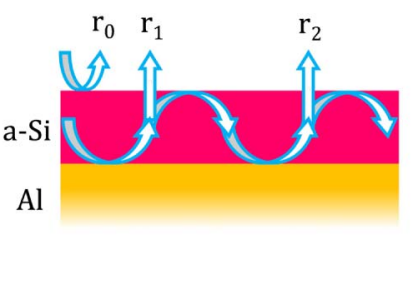

(d)

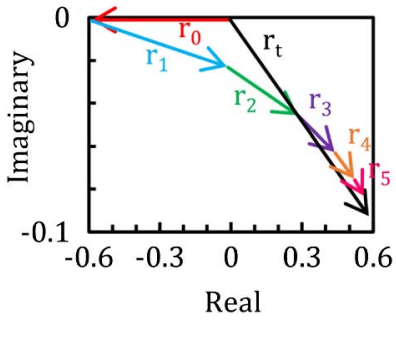

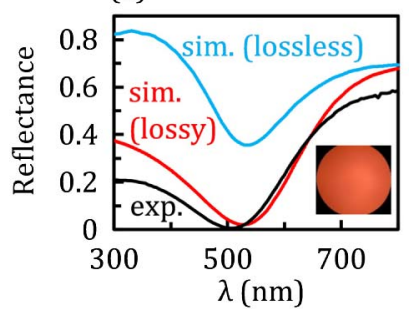

Fig. 1. (a) Refractive indices and extinction coefficients of amorphous silicon and aluminum used in the simulations. (b) Cross-sectional illustration of the reflected light rays from a-Si/Al surface. (c) Simulated reflection coefficients on the complex plane for $20 \mathrm{~nm}$ thick a-Si on Al at $\lambda=526 \mathrm{~nm}$. (d) Simulated reflection coefficients for $20 \mathrm{~nm}$ thick a-Si (assuming $k=0$ ) on $\mathrm{Al}$ on the complex plane at $\lambda=534 \mathrm{~nm}$. (e) Measured reflection spectrum and simulation results using the extinction coefficient of a-Si (lossy) and zero extinction coefficient (lossless). Inset: microscope image of the surface.

the destructive interference of the reflected rays, the reflection coefficients are plotted on the complex plane at the wavelength at which the minimum reflectance is observed. For $20 \mathrm{~nm}$ thick a-Si on $\mathrm{Al}$, all of the reflection coefficients are complex owing to the complex refractive indices of a-Si and Al [Fig. 1(c)]. For a complete destructive interference, the vector sum of all the reflection coefficients $\left(r_{t}\right)$ must be zero. For the studied case, $r_{t}$ is very small $(-0.096-0.113 i)$, resulting in a small reflectance $\left(\left|r_{t}\right|^{2}=0.022\right)$ at the given wavelength. As an exercise, the reflection coefficients are calculated assuming a zero extinction coefficient for a-Si. In this case, the destructive interference is still present, but weaker, resulting in a larger total reflectance [Fig. 1(d)]. Note that $r_{0}$ is a real number owing to the real refractive indices of air and the dielectric. The total reflection coefficient can analytically be calculated as

$$
r_{t}=\frac{r_{0}+r_{12} e^{2 i \beta}}{1+r_{0} r_{12} e^{2 i \beta}},
$$

where $\beta$ is the complex phase accumulation in the light traveling in the a-Si layer, and $r_{12}$ is the reflection coefficient for the a-Si-metal interface that is calculated as $\left(n_{1}-n_{2}\right) /\left(n_{2}+n_{1}\right)$ for the normal incidence, where $n_{1}$ and $n_{2}$ are the complex refractive indices of a-Si and metal, respectively. As a result, a thin

lossy dielectric layer is required on a metal mirror to achieve strong absorbance (strong interference effects) around a certain wavelength and to create distinct surface colors [Fig. 1(e)]. Equation (1) shows that $\beta$, hence $r_{t}$, changes with increasing a-Si thickness, determining the reflection spectrum and the surface color. The measured reflectance spectrum shows almost perfect absorbance $(99.5 \%)$ at $\lambda=505 \mathrm{~nm}$, resulting in a surface color containing mainly red [Fig. 1(e)]. The small discrepancy between the simulated and measured reflection spectra is attributed to the surface roughness of the Al films that is estimated as $2 \mathrm{~nm}$ from atomic force microscopy measurements. The surfaces are imaged under a microscope with $5 \times$ magnification as they are illuminated by a halogen lamp to keep the illumination condition consistent for all the surfaces.

Recently, various strong interference coatings have been demonstrated using lossy dielectrics such as a-Si [33], Ge [34], $\mathrm{Ge}_{2} \mathrm{Sb}_{2} \mathrm{Te}_{5}$ [35], and $\mathrm{VO}_{2}$ [36]. Although Ge is used as the dielectric layer for the color printing applications, it suffers from degradation in ionic solutions; hence, it is not suitable for bio-sensing applications. In this Letter, we have used $\mathrm{a}-\mathrm{Si}$ and $\mathrm{Al}$ to fabricate strong interference coatings due to the abundance and complementary metal-oxide-semiconductor (CMOS) compatibility of both materials. Moreover, a-Si/Al surfaces provide enhanced color contrast when coated with ultrathin dielectrics compared to the alternatives such as a-Si/Ag. The surfaces are fabricated using thermal evaporation. The optical properties of the materials are extracted using variableangle spectroscopic ellipsometry (J. A. Woollam V-Vase). The refractive indices and extinction coefficients of $\mathrm{Al}$ and a-Si are shown in Fig. 1(a), where Drude-Lorentz and Tauc-Lorentz models are used for $\mathrm{Al}$ and a-Si, respectively. The thickness of the a-Si layer is chosen to achieve an absorption peak (minimum reflectance) in the middle of the visible wavelength range $(\sim 530 \mathrm{~nm})$ [Fig. 1(e)]. Figures 2(a) and 2(b) show that the reflection spectra are undisturbed up to extreme angles of incidence for both $s$ - and $p$-polarization. Angle- and polarization-independent reflection spectra have been reported previously for other strong interference surfaces and are attributed to small phase accumulation in the reflected light for any angle of incidence owing to ultrathin coating layers. Such an omnidirectional optical response is especially preferred for the application proposed here as any light source, polarization, and viewing angle is acceptable.

For a thin lossless dielectric layer on top of a strong interference surface, the total reflection coefficient can be calculated as follows: (a) Reflectance

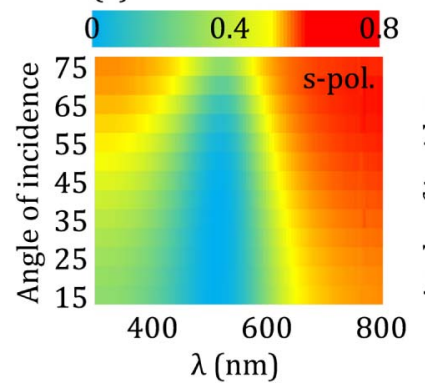

(b) Reflectance

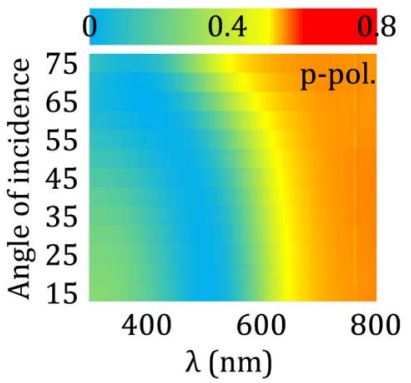

Fig. 2. Measured reflection spectra for (a) $s$ - and (b) $p$-polarization and varying angles of incidence. 


$$
r_{t}=\frac{r_{0}+r_{a-\mathrm{Si} / \mathrm{Al}} e^{2 i \beta}}{1+r_{0} r_{a-\mathrm{Si} / \mathrm{Al}} e^{2 i \beta}},
$$

where $r_{0}$ is the reflection coefficient for the air-dielectric interface, $r_{a-\mathrm{Si} / \mathrm{Al}}$ is the reflection coefficient from the strong interference surface that is $r_{t}$ in Eq. (1), and $\beta$ is the phase accumulation in the light traveling in the dielectric layer. Since $\beta=2 \pi n_{1} t / \lambda$, where $n_{1}$ and $t$ are the refractive index and the thickness of the dielectric layer, $r_{t}$ in Eq. (2), hence, the reflectance spectrum, is altered changing the surface color for varying dielectric thicknesses. The wide-angle performance of the bare strong interference surfaces is undisturbed after coating nanometer-thick lossless dielectrics.

The sensing performance of the strong interference surfaces is further characterized by depositing lossless dielectric films, namely $\mathrm{SiO}_{2}$ and $\mathrm{Al}_{2} \mathrm{O}_{3}$, with varying thicknesses $(5-20 \mathrm{~nm})$. Such dielectric films have refractive indices similar to those of protein films and, thus, can be used to characterize the surface sensitivities of optical bio-sensors. The thicknesses and refractive indices of the deposited films are characterized by variableangle spectroscopic ellipsometry measurements. $\mathrm{SiO}_{2}$ films are deposited using e-beam evaporation where $\mathrm{Al}_{2} \mathrm{O}_{3}$ films are deposited using atomic layer deposition. The absorption peak red shifts for increasing $\mathrm{SiO}_{2}$ and $\mathrm{Al}_{2} \mathrm{O}_{3}$ thicknesses [Figs. 3(a) and $3(\mathrm{~b})$ ]. The corresponding color shift is visible in the optical microscope images [Figs. 3(c) and 3(d)]. The analysis of the red-green-blue (RGB) components of the photographs taken under a halogen lamp shows that the blue channel intensity
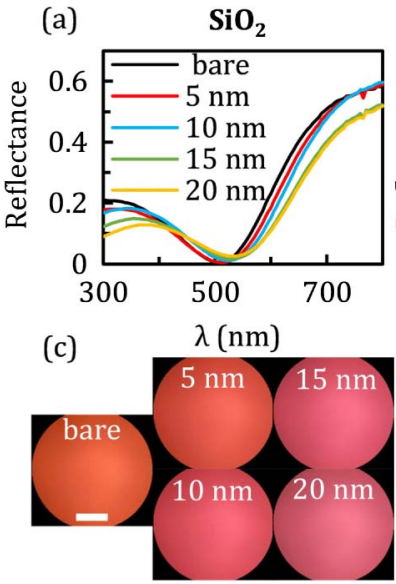

(e)

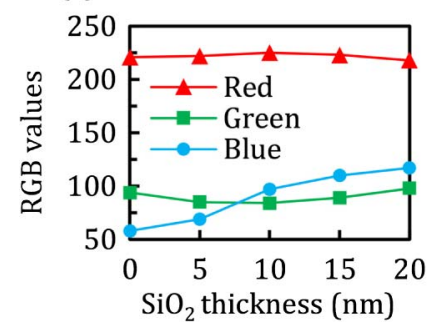

(b)

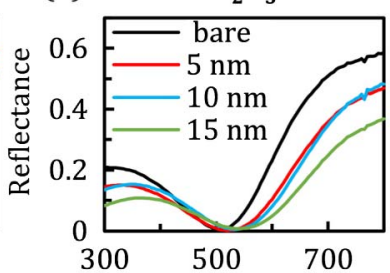

(d)

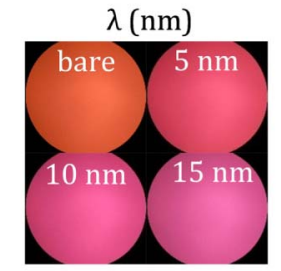

(f)

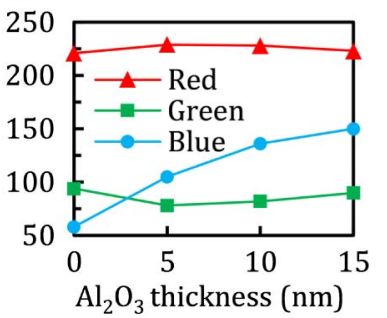

Fig. 3. (a) Measurement results for nanometer-thick $\mathrm{SiO}_{2}$ and $\mathrm{Al}_{2} \mathrm{O}_{3}$ films on $20 \mathrm{~nm}$ a-Si on Al. (a), (b) Reflection spectra for increasing $\mathrm{SiO}_{2}$ and $\mathrm{Al}_{2} \mathrm{O}_{3}$ thicknesses. (c), (d) Photographs of the surfaces under microscope illuminated by a halogen lamp. $\mathrm{SiO}_{2}$ and $\mathrm{Al}_{2} \mathrm{O}_{3}$ thicknesses are shown on the images. The scale bar in (c) represents $1 \mathrm{~mm}$. (e), (f) Corresponding RGB values of the surfaces for increasing $\mathrm{SiO}_{2}$ and $\mathrm{Al}_{2} \mathrm{O}_{3}$ thicknesses. increases with increasing $\mathrm{SiO}_{2}$ and $\mathrm{Al}_{2} \mathrm{O}_{3}$ thicknesses, while the green and red channel intensities are almost constant [Figs. 3(e) and 3(f)]. The change in the blue channel intensity is attributed to the red shift in the absorption peak, where the blue portion (400-450 nm) of the reflection spectrum increases with the $\mathrm{SiO}_{2}$ and $\mathrm{Al}_{2} \mathrm{O}_{3}$ thicknesses. The shift in the absorption peak is greater for $\mathrm{Al}_{2} \mathrm{O}_{3}$ owing to its larger refractive index. For instance, for the surface coated with $15 \mathrm{~nm}$ thick $\mathrm{Al}_{2} \mathrm{O}_{3}$, the overall shift is $40 \mathrm{~nm}$ which is larger than the $25 \mathrm{~nm}$ shift observed for $15 \mathrm{~nm}$ thick $\mathrm{SiO}_{2}$. As a result, the change in the surface color is more enhanced for $\mathrm{Al}_{2} \mathrm{O}_{3}$ coating compared to $\mathrm{SiO}_{2}$ coating with the same thickness.

After verifying the color changes due to nanometer-thick dielectric layers, we utilized the same surfaces as colorimetric detection of protein monolayer and bilayer films. Colorimetric biosensor surfaces have been demonstrated recently using plasmonic nanostructured surfaces [37-40]. In these studies, different refractive index liquids, monolayer protein films, and antibody-antigen interactions have been detected as color or intensity change. In another study, the colorimetric detection of nucleic acids has been demonstrated using $\mathrm{SiN}_{x}$ on $\mathrm{Si}$ interference coatings [41]. Here, the bio-sensing performance of the surfaces is tested with monolayer and bilayer bovine serum albumin (BSA) protein molecules. BSA molecules are commonly employed for bio-sensing studies owing to their high affinity for a variety of surfaces such as oxides [42,43]. BSA monolayers are achieved by physical adsorption on the surfaces. Bilayers are achieved after treating the BSA-coated surfaces with dextrane [Fig. 4(a)]. The absorption peak red shifts by $\sim 8 \mathrm{~nm}$ for monolayer BSA and by $\sim 18 \mathrm{~nm}$ for dextrane and another layer of BSA [Fig. 4(b)]. The strong shifts in the reflection spectrum result in noticeable surface color changes [Figs. 4(c) and 4(d)].

It is worth noting that no change in the optical response is observed for the surfaces that are exposed to the citrate solution

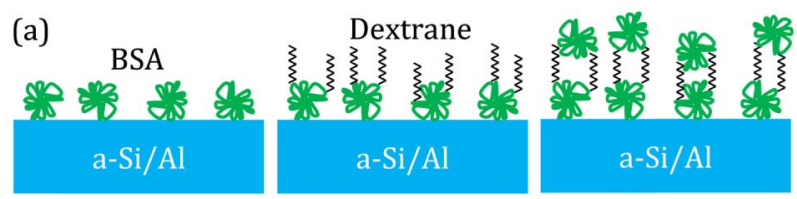

(b)

(c)
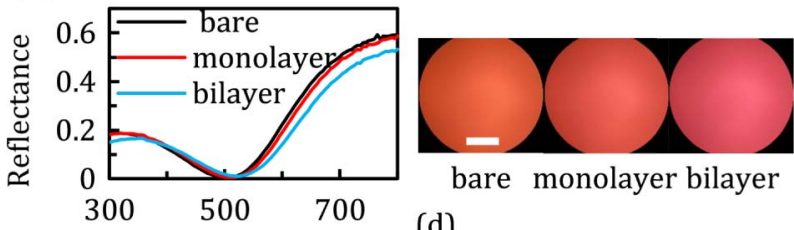

(d)

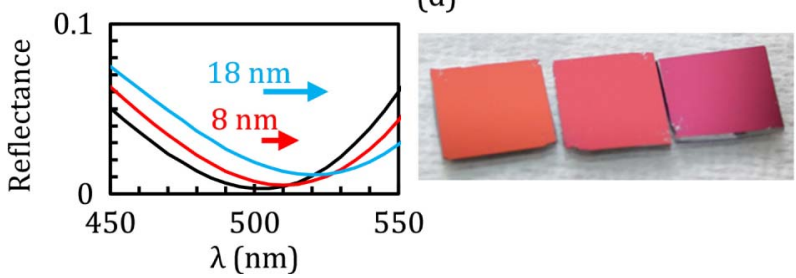

Fig. 4. Protein binding experiments. (a) Illustration of monolayer and bilayer BSA formation on the sensor surface. (b) Measured reflection spectra of surfaces without monolayer and bilayer protein molecules. Photographs of the surfaces (c) taken under a microscope with $5 \times$ magnification illuminated with a halogen lamp and (d) taken under ambient light. The scale bar in (c) represents $1 \mathrm{~mm}$. 
without BSA. Thus, it is concluded that the results summarized in Fig. 4 are due to BSA adsorption on the surfaces, and not due to any other physical or chemical changes on the surfaces. Monolayer BSA formation on the a-Si is also confirmed via IR absorption spectroscopy using $15 \mathrm{~nm}$ a-Si-coated fieldenhancement surfaces based on $0.9 \mu \mathrm{m}$ thick $\mathrm{CaF}_{2}$ on $\mathrm{Al}$. Such surfaces have previously applied for enhanced IR absorption spectroscopy of monolayers of BSA on $\mathrm{CaF}_{2}$ surfaces [28]. The $\mathrm{CaF}_{2}$ surface is covered with a thin a-Si layer to verify the affinity of BSA molecules to the a-Si film.

In summary, we demonstrate a colorimetric sensor platform based on strong interference effects using $20 \mathrm{~nm}$ thick a-Si on optically thick Al films. The bare surfaces appear in a color containing mostly red. Small changes on the a-Si surface such as the deposition of nanometer-thick dielectric layers cause the absorption peak to red shift, increase the blue portion of the reflection spectrum, and change the surface colors. The surfaces exhibit similar color changes when coated with monolayer and bilayer BSA molecules, thus allowing the detection of these layers by the naked eye. The strong interference surfaces consist of patternless thin metal and lossy dielectric layers and, hence, are low in cost and easy to fabricate on large areas. Such surfaces have the potential for bio-sensing applications.

\section{REFERENCES}

1. J. Valentine, S. Zhang, T. Zentgraf, E. Ulin-Avila, D. A. Genov, G. Bartal, and X. Zhang, Nature 455, 376 (2008).

2. T. Xu, A. Agrawal, M. Abashin, K. J. Chau, and H. J. Lezec, Nature 497, 470 (2013)

3. N. Landy, S. Sajuyigbe, J. Mock, D. Smith, and W. Padilla, Phys. Rev. Lett. 100, 207402 (2008).

4. C. M. Watts, X. Liu, and W. J. Padilla, Adv. Mater. 24, OP98 (2012).

5. A. Arbabi, Y. Horie, A. J. Ball, M. Bagheri, and A. Faraon, Nat. Commun. 6, 7069 (2015).

6. M. Khorasaninejad, W. T. Chen, R. C. Devlin, J. Oh, A. Y. Zhu, and F. Capasso, Science 352, 1190 (2016).

7. R. F. Oulton, V. J. Sorger, D. A. Genov, D. F. P. Pile, and X. Zhang, Nat. Photonics 2, 496 (2008).

8. Y. Fang and M. Sun, Light Sci. Appl. 4, e294 (2015).

9. A. E. Cetin, A. F. Coskun, B. C. Galarreta, M. Huang, D. Herman, A. Ozcan, and H. Altug, Light Sci. Appl. 3, e122 (2014).

10. D. Dey, J. Kohoutek, R. M. Gelfand, A. Bonakdar, and H. Mohseni, Opt. Lett. 35, 2783 (2010).

11. C. Shemelya, D. DeMeo, N. P. Latham, X. Wu, C. Bingham, W. Padilla, and T. E. Vandervelde, Appl. Phys. Lett. 104, 201113 (2014).

12. X. Liu, T. Tyler, T. Starr, A. F. Starr, N. M. Jokerst, and W. J. Padilla, Phys. Rev. Lett. 107, 45901 (2011).

13. N. Liu, M. Mesch, T. Weiss, M. Hentschel, and H. Giessen, Nano Lett. 10, 2342 (2010).

14. A. Tittl, P. Mai, R. Taubert, D. Dregely, N. Liu, and H. Giessen, Nano Lett. 11, 4366 (2011).
15. K. Kumar, H. Duan, R. S. Hegde, S. C. W. Koh, J. N. Wei, and J. K. W. Yang, Nat. Nanotechnol. 7, 557 (2012).

16. A. Kristensen, J. K. W. Yang, S. I. Bozhevolnyi, S. Link, P. Nordlander, N. J. Halas, and N. A. Mortensen, Nat. Rev. Mater. 2, 16088 (2016).

17. X. Liu, T. Starr, A. F. Starr, and W. J. Padilla, Phys. Rev. Lett. 104, 207403 (2010).

18. J. Hendrickson, J. Guo, B. Zhang, W. Buchwald, and R. Soref, Opt. Lett. 37, 371 (2012).

19. K. Aydin, V. E. Ferry, R. M. Briggs, and H. A. Atwater, Nat. Commun. 2, 517 (2011).

20. F. Wang and N. A. Melosh, Nat. Commun. 4, 1711 (2013).

21. Z. Yang, Y. Zhou, Y. Chen, Y. Wang, P. Dai, Z. Zhang, and H. Duan, Adv. Opt. Mater. 4, 1196 (2016).

22. H. Kwon and S. Kim, ACS Photon. 2, 1675 (2015).

23. Z. Li, E. Palacios, S. Butun, H. Kocer, and K. Aydin, Sci. Rep. 5, 15137 (2015).

24. X. Duan, S. Kamin, and N. Liu, Nat. Commun. 8, 14606 (2017).

25. P. Ngene, T. Radeva, M. Slaman, R. J. Westerwaal, H. Schreuders, and B. Dam, Adv. Funct. Mater. 24, 2374 (2014).

26. H. Song, L. Guo, Z. Liu, K. Liu, X. Zeng, D. Ji, N. Zhang, H. Hu, S. Jiang, and Q. Gan, Adv. Mater. 26, 2737 (2014).

27. Z. Chen, L. Zhu, A. Raman, and S. Fan, Nat. Commun. 7, 13729 (2016).

28. S. Ayas, G. Bakan, E. Ozgur, K. Celebi, and A. Dana, ACS Photon. 3, 337 (2016).

29. M. A. Kats, R. Blanchard, P. Genevet, and F. Capasso, Nat. Mater. 12, 20 (2013).

30. H. Dotan, O. Kfir, E. Sharlin, O. Blank, M. Gross, I. Dumchin, G. Ankonina, and A. Rothschild, Nat. Mater. 12, 158 (2013).

31. M. A. Kats, S. J. Byrnes, R. Blanchard, M. Kolle, P. Genevet, J. Aizenberg, and F. Capasso, Appl. Phys. Lett. 103, 101104 (2013).

32. F. F. Schlich, P. Zalden, A. M. Lindenberg, and R. Spolenak, ACS Photon. 2, 178 (2015).

33. K.-T. Lee, C. Ji, and L. J. Guo, Appl. Phys. Lett. 108, 31107 (2016).

34. S. J. Kim, P. Fan, J.-H. Kang, and M. L. Brongersma, Nat. Commun. 6, 7591 (2015).

35. G. Bakan, S. Ayas, T. Saidzoda, K. Celebi, and A. Dana, Appl. Phys. Lett. 109, 071109 (2016).

36. M. A. Kats, D. Sharma, J. Lin, P. Genevet, R. Blanchard, Z. Yang, M. M. Qazilbash, D. N. Basov, S. Ramanathan, and F. Capasso, Appl. Phys. Lett. 101, 221101 (2012).

37. M. R. Gartia, A. Hsiao, A. Pokhriyal, S. Seo, G. Kulsharova, B. T. Cunningham, T. C. Bond, and G. L. Liu, Adv. Opt. Mater. 1, 1 (2013).

38. M. Khorasaninejad, S. Mohsen Raeis-Zadeh, H. Amarloo, N. Abedzadeh, S. Safavi-Naeini, and S. S. Saini, Nanotechnology 24 355501 (2013).

39. S. Y. Lee, J. J. Amsden, S. V. Boriskina, A. Gopinath, A. Mitropolous, D. L. Kaplan, F. G. Omenetto, and L. Dal Negro, Proc. Natl. Acad. Sci. USA 107, 12086 (2010).

40. N. S. King, L. Liu, X. Yang, B. Cerjan, H. O. Everitt, P. Nordlander, and N. J. Halas, ACS Nano 9, 10628 (2015).

41. R. Jenison, S. Yang, A. Haeberli, and B. Polisky, Nat. Biotechnol. 19, 62 (2001).

42. M. Piliarik and J. Homola, Opt. Express 17, 16505 (2009).

43. P. I. Nikitin, B. G. Gorshkov, E. P. Nikitin, and T. I. Ksenevich, Sens. Actuators B 111, 500 (2005). 